\title{
EXAMPLES OF LOCALLY TRIVIAL AZUMAYA ALGEBRAS
}

\author{
T. J. FORD
}

\begin{abstract}
An Azumaya algebra $A$ on a variety $X$ that splits at every point of $X$ is a maximal order in a matrix algebra over the function field, hence is the endomorphism ring of a coherent sheaf $M$ of reflexive modules on $X$. Many examples of such algebras exist in the literature but they all rely on nonconstructive existence proofs. Several concrete examples of such algebras are presented. The rank of the module $M$ is immediately known in each case. For certain varieties $X$, our technique allows us to construct algebras $A$ which were not previously known to exist. A procedure is given for computing both the Picard group and the cohomological Brauer group of any toric variety.
\end{abstract}

\section{INTRODUCTION}

Let $X$ be a variety over the algebraically closed field $k$ of characteristic 0 . The Brauer group of $X$, denoted $\mathrm{B}(X)$, parameterizes the classes of Azumaya $\mathcal{O}_{X^{-}}$ algebras on $X$ [16, Chap. IV]. If $K$ denotes the function field of $X$, then the kernel of the natural map $\mathrm{B}(X) \rightarrow \mathrm{B}(K)$ is denoted by $\mathrm{B}(K / X)$. An Azumaya $\mathcal{O}_{X}$-algebra $A$ whose class is in $B(K / X)$ is a maximal order in a matrix algebra over $K$, hence is the endomorphism ring $\operatorname{End}_{\mathcal{O}_{X}}(M)$ for some coherent sheaf of reflexive modules $M$ on $X$ [2], [15].

From now on suppose $X$ is a normal variety. In this note we consider the kernel of the natural homomorphism

$$
\theta: \mathrm{B}(X) \rightarrow \prod_{p \in X} \mathrm{~B}\left(\mathcal{O}_{p}\right) .
$$

This kernel consists of algebra classes $[A]$ where $A$ is an Azumaya algebra such that at every point $p \in X, A_{p}$ is isomorphic to the endomorphism ring $\operatorname{End}_{\mathcal{O}_{p}}(F)$ of a free $\mathcal{O}_{p}$-module $F$ (see [1]). As mentioned above, $A \cong \operatorname{End}_{\mathcal{O}_{X}}(M)$ for a reflexive module $M$ on $X$. It follows from [1], [15] that there is an open affine cover $\left\{U_{j}\right\}$ of $X$ such that for each $j,\left.M\right|_{U_{j}} \cong I_{j} \otimes F_{j}$ where $I_{j}$ is a rank 1 reflexive module on $U_{j}$ and $F_{j}$ is a free module on $U_{j}$. For a survey of the study of nontrivial locally trivial algebras, see [6].

1991 Mathematics Subject Classification. 16Ho5, secondary 13A20, 14F20, 14-04.

Supported in part by the N.S.F. under grant DMS-9025092.

K-theory and Algebraic Geometry: Connections with Quadratic Forms and Division Algebras (Santa Barbara, CA, 1992), Part 2, vol. 58 of Proc. Sympos. Pure Math., pp. 197-216

(C)1995, American Mathematical Society, Providence, RI.. 
By [ 1 , Theorem 1$]$ if $X$ is affine and by [15, Theorem 5.1(c)] in general, there is an exact sequence of groups

$$
0 \rightarrow \operatorname{Pic} X \rightarrow \mathrm{Cl}(X) \rightarrow \mathrm{BCl}\left(\mathbf{P}, \mathbf{I}_{X}\right) \rightarrow \mathrm{B}(X) \stackrel{\theta}{\rightarrow} \prod_{p \in X} \mathrm{~B}\left(\mathcal{O}_{p}\right) .
$$

The group $\mathrm{BCl}\left(\mathbf{P}, \mathbf{I}_{X}\right)$ is the set of classes of coherent reflexive $\mathcal{O}_{X}$-modules $M$ such that for each $p \in X, M_{p} \cong I_{p} \otimes F_{p}$ for a rank 1 reflexive $\mathcal{O}_{p}$-module $I_{p}$ and a free $\mathcal{O}_{p}$-module $F_{p}$. Two such $M_{1}$ and $M_{2}$ belong to the same class in $\operatorname{BCl}\left(\mathbf{P}, \mathbf{I}_{X}\right)$ if there exist coherent locally free $\mathcal{O}_{X}$-modules $N_{1}$ and $N_{2}$ such that $\left(M_{1} \otimes N_{1}\right)^{* *} \cong\left(M_{2} \otimes N_{2}\right)^{* *}$. The group operation is induced by tensoring and taking the double dual. The inverse class of $M$ is the class of the dual of $M$. The homomorphism $\mathrm{BCl}\left(\mathbf{P}, \mathbf{I}_{X}\right) \rightarrow \mathrm{B}(X)$ sends the class of $M$ to $\operatorname{End}_{\mathcal{O}_{X}}(M)$.

As in $\left[7\right.$, Lemma 4] let $\mathcal{C}$ denote the sheaf of Cartier divisors on $X_{Z \text { Zar }}$ and $\mathcal{W}$ the sheaf of Weil divisors. There is an exact sequence of sheaves on $X_{Z a r}$

$$
0 \rightarrow \mathcal{C} \rightarrow \mathcal{W} \rightarrow \mathcal{P} \rightarrow 0
$$

which defines the sheaf $\mathcal{P}$. Because every locally principal Weil divisor is in $\mathcal{C}$, we see that $\mathcal{P}$ is the sheaf associated to $\mathrm{Cl}(\cdot)$. Because $\mathcal{W}$ is flasque, there is an exact sequence of cohomology groups associated to (2)

(3) $0 \rightarrow H^{0}\left(X_{\mathrm{Zar}}, \mathcal{C}\right) \rightarrow H^{0}\left(X_{\mathrm{Zar}}, \mathcal{W}\right) \rightarrow H^{0}\left(X_{\mathrm{Zar}}, \mathcal{P}\right) \rightarrow H^{1}\left(X_{\mathrm{Zar}}, \mathcal{C}\right) \rightarrow 0$

Because a principal Weil divisor is a principal Cartier divisor [11, II.6.11.2], (3) gives rise to

$$
0 \rightarrow \operatorname{Pic} X \rightarrow \mathrm{Cl}(X) \rightarrow H^{0}\left(X_{\mathrm{Zar}}, \mathcal{P}\right) \rightarrow H^{1}\left(X_{\mathrm{Zar}}, \mathcal{C}\right) \rightarrow 0 .
$$

As in [1] there is a monomorphism $\alpha: \operatorname{BCl}\left(\mathbf{P}, \mathbf{I}_{X}\right) \rightarrow H^{0}\left(X_{Z a r}, \mathcal{P}\right)$ defined by sending the class of $M$ to the global cross-section of $\mathcal{P}$ whose value at the stalk of $p \in X$ is $I_{p}$, where $M_{p} \cong I_{p} \otimes F_{p}$ as above. This gives a commutative diagram whose rows are (4) and (I).

(5)

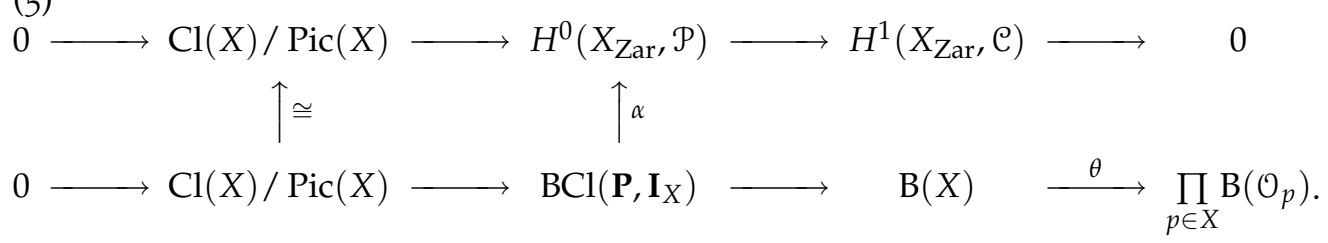


The Brauer group of a variety $X$ is studied by means of the canonical embedding

$$
i: \mathrm{B}(X) \rightarrow \operatorname{tors}\left(H^{2}\left(X_{\text {ét }}, \mathbb{G}_{m}\right)\right)
$$

into the cohomological Brauer group which we denote by $\mathrm{B}^{\prime}(X)$. For a survey of results concerning the surjectivity of $i$ see [12]. For example, the HooblerGabber Theorem says that $i$ is surjective when $X$ is an affine scheme [12, Theorem 7]. It follows from cohomology theory (see [10, II], [6]) that $H^{2}\left(K / X_{\text {ét }}, \mathbb{G}_{m}\right) \cong$ $H^{1}\left(X_{\text {ét }}, \mathcal{C}\right)$ where $\mathcal{C}$ is the sheaf of Cartier divisors on $X_{\text {ét }}$ and also, since $X$ is normal, that the sequence [10, I,II]

$$
\mathrm{Cl}(X) \rightarrow H^{0}\left(X_{\text {ét }}, \mathcal{P}\right) \rightarrow H^{1}\left(X_{\text {ét }}, \mathcal{e}\right) \rightarrow 0
$$

is exact where $\mathrm{Cl}(X)$ is the group of Weil divisor classes and $\mathcal{P}$ is the sheaf associated to the functor $\mathrm{Cl}(\cdot)$ on $X_{\text {ét. }}$. Using these cohomological results, it is possible to compute $\mathrm{B}^{\prime}(K / X)$ for many choices of $X$ (e.g. [9], [6], [7], and Section 4). To translate these computations into statements about Azumaya algebras, one is almost always forced to quote the Hoobler-Gabber Theorem in its full force.

The proof that $i$ is surjective, as given in [12], first reduces the problem down to the locally trivial case. This is an important reason to study locally trivial Azumaya algebras.

The proof of the Hoobler-Gabber Theorem is based on Quillen induction which allows one to pass from local to global results on a variety which is the separated union of 2 affine varieties $U_{1} \cup U_{2}$. A key step in the proof uses a Mayer-Vietories sequence

$$
\operatorname{Pic} U_{1} \oplus \operatorname{Pic} U_{2} \rightarrow \operatorname{Pic}\left(U_{1} \cap U_{2}\right) \stackrel{\partial}{\rightarrow} H^{2}\left(U_{1} \cup U_{2}, G_{m}\right)
$$

to show that any cohomology class $y$ in $\mathrm{B}^{\prime}\left(U_{1} \cup U_{2}\right)$ that is trivial on $U_{1}$ and $U_{2}$ is in $\mathrm{B}\left(U_{1} \cup U_{2}\right)$. The proof is based on the stability theorems of Bass [4] and shows that $y$ is the class of $\operatorname{End}(M)$ for some reflexive module $M$.

Unfortunately the proof of the existence of $M$ does not supply us with a lot of specific information. For example, the rank of $M$ is usually not known and even though the local structure of $M$ is given, the 1-cocycle that shows how to patch the local models together is obscure. There exist bounds for $\operatorname{rank}(M)$ (see [3]) but even in some of the simplest examples it has not been computed exactly (see, e.g., [6], [8]). All known examples in the literature (see, e.g. [18], [6], [8]) of locally trivial Azumaya algebras have relied on the above method to show the existence of $M$. For this reason we feel it is desirable to have some concrete examples of such modules $M$. 
In this note we present a number of examples of normal integral varieties $X$ and a totally elementary description of reflexive modules $M$ on $X$ such that End $(M)$ represents a nontrivial class in $\mathrm{B}(X)$. As a result of our construction, the rank of $M$ is immediately known in each case. We are able to show that $\mathrm{B}(X)=\mathrm{B}^{\prime}(X)$ in some examples where it was previously unknown because the Hoobler-Gabber Theorem could not be directly applied.

In Section 4, we describe a procedure for computing the lower degree étale cohomology groups of a toric variety with coefficients in the sheaf of units. The procedure is based on recent results in [7].

\section{The EXAMples}

Let $X$ be a normal variety and $A=\operatorname{End}_{\mathcal{O}_{X}}(M)$ an algebra on $X$ whose Brauer class is in the kernel of $\theta$. From Section 1 we know that $X$ has an affine open cover $\left\{U_{j}\right\}$ and $\left.M\right|_{U_{j}} \cong I_{j} \otimes F_{j}$ where $I_{j}$ is a reflexive module of rank $I$ and $F_{j}$ is free. If $\operatorname{Sing}\left(U_{j}\right)$ denotes the singular locus of $U_{j}$, then on $\left(U_{j}\right)_{\text {reg }}=U_{j}-\operatorname{Sing}\left(U_{j}\right)$, $\left.I_{j}\right|_{\left(U_{j}\right)_{\text {reg }}}$ is an invertible module. If $g:\left(U_{j}\right)_{\text {reg }} \rightarrow U_{j}$ is the open immersion, then $g_{*} g^{*} I \cong I$ and $\left.\left.g_{*} g^{*} M\right|_{U_{j}} \cong M\right|_{U_{j}}$ since $\left(U_{j}\right)_{\text {reg }}$ contains all of the points of codimension 1 . So to construct $M$ it suffices to construct a coherent locally free sheaf $P$ on $X_{\text {reg }}=\cup\left(U_{j}\right)_{\text {reg }}$ such that $\left.P\right|_{\left(U_{j}\right)_{\text {reg }}} \cong\left(I_{j} \otimes F_{j}\right) \mid\left(U_{j}\right)_{\text {reg. }}$. (In fact, it is enough to construct $P$ on any open subset $W$ of $X_{\text {reg }}$ such that $W$ contains all of the points of codimension 1. We use this fact in Examples 2.4 and 2.5. The isomorphism classes of locally free $\mathcal{O}_{X_{\text {reg }}}$-modules of rank $n$ are parametrized by $\breve{H}^{1}\left(X_{\text {reg }}, G L_{n}\right)$ [16, p. 134]. So to specify $P$ it suffices to find an open cover $\left\{V_{k}\right\}$ of $X_{\text {reg }}$ and a Čech 1-cocycle $\left\{\phi_{i j} \in G L_{n}\left(V_{i} \cap V_{j}\right)\right\}$ satisfying:

(1) the 1-cocycle identities: for each $i, j, k, \phi_{i k}=\phi_{j k} \phi_{i j}$, and

(2) the restriction of $\left\{\phi_{i k}\right\}$ to $\left(U_{j}\right)_{\text {reg }}$ defines the locally free sheaf $\left.\left(I_{j} \otimes F_{j}\right)\right|_{\left(U_{j}\right)_{\text {reg }}}$.

Example 2.1. Let $n$ equal 2 or 3 . This is an example of an affine normal surface $X=\operatorname{Spec} R$ such that $\mathrm{B}(K / R)=\mathbb{Z} / n$. Let $l_{1}, l_{2}, l_{3}$ be polynomials in $k[x, y]$ that generate the unit ideal. Also assume that for each pair $l_{i}, l_{j}$ the ideal $\left(l_{i}, l_{j}\right)$ is maximal. In other words we are assuming the curve defined by the equation $l_{1} l_{2} l_{3}=0$ has exactly 3 ordinary double points and the 3 irreducible components $L_{1}, L_{2}, L_{3}$ of this curve satisfy $L_{i} \cdot L_{j}=1$ for each pair $l_{i}, l_{j}$. Let

$$
R=\frac{k[x, y, z]}{\left(z^{n}-l_{1} l_{2} l_{3}\right)} .
$$


As was computed in [6, Example 3], $\mathrm{B}(K / R) \cong \mathbb{Z} / n$ and every Azumaya algebra on $X=\operatorname{Spec} R$ is split by the affine open cover $\left\{U_{i}=\operatorname{Spec}\left(R_{l_{i}}\right)\right\}$. Let $p_{i}$ denote the unique singular point in $U_{i}$. Then $\mathrm{Cl}\left(U_{i}\right) \cong \operatorname{Pic}\left(U_{i}-p_{i}\right)$. Also by [6] $\mathrm{Cl}\left(U_{i}\right) / \operatorname{Pic}\left(U_{i}\right) \cong \mathrm{Cl}\left(\mathcal{O}_{p_{i}}\right) \cong \mathbb{Z} / n, H^{0}\left(X_{\text {Zar }}, \mathcal{P}\right) \cong H^{0}\left(X_{\text {ét }}, \mathcal{P}\right)$ and therefore by (4) and (6) $H^{1}\left(X_{\mathrm{Zar}}, \mathcal{C}\right) \cong \mathrm{B}(K / R)$. We have an affine open cover for $X-p_{1}-p_{2}-p_{3}$, namely $V_{1}=\operatorname{Spec}\left(R_{l_{2} l_{3}}\right), V_{2}=\operatorname{Spec}\left(R_{l_{1} l_{3}}\right), V_{3}=\operatorname{Spec}\left(R_{l_{1} l_{2}}\right)$. Note that $V_{1} \cap V_{2}=V_{1} \cap V_{3}=V_{2} \cap V_{3}=\operatorname{Spec}\left(R_{z}\right)$. Also $U_{1}-p_{1}=V_{2} \cup V_{3}$, $U_{2}-p_{2}=V_{1} \cup V_{3}, U_{3}-p_{3}=V_{1} \cup V_{2}$.

Our plan is to construct a reflexive module $M$ of rank $n$ on $X$ such that $\left.M\right|_{U_{3}} \cong$ $n I(=I \oplus \ldots \oplus I, n$ times), where $I$ is a reflexive ideal that is a generator for $\mathrm{Cl}\left(\mathcal{O}_{p_{3}}\right)$. Also we build $M$ so that $\left.M\right|_{U_{1}}$ is free and $\left.M\right|_{U_{2}}$ is free. Then from (5) we know that $\operatorname{End}_{R}(M)$ represents a nontrivial class in $\mathrm{B}(R)$ with exponent $n$.

Since $\operatorname{Cl}\left(U_{3}\right) \cong \operatorname{Pic}\left(U_{3}-p_{3}\right)=\operatorname{Pic}\left(V_{1} \cup V_{2}\right)$ it suffices to construct a vector bundle $P$ on $V_{1} \cup V_{2} \cup V_{3}$ of rank $n$ such that on $V_{1} \cup V_{2}, P \cong n J$ where $J$ is the invertible module $\left.I\right|_{V_{1} \cup V_{2}}$. On both $V_{1} \cup V_{3}$ and $V_{2} \cup V_{3}$ we want $P$ to be free. The local model for $P$ on each $V_{i}$ will be $n \mathcal{O}$. It remains to find patching isomorphisms $\phi_{i j}$ in $G L_{n}\left(V_{1} \cap V_{2} \cap V_{3}\right)=G L_{n}(R[1 / z])$ which satisfy the 1-cocycle identity: $\phi_{12}=\phi_{32} \phi_{13}$. We choose $\phi_{12}$ so that $\left.P\right|_{V_{1} \cup V_{2}} \cong n \mathrm{~J}$. We choose $\phi_{32}$ and $\phi_{13}$ to be coboundaries from $V_{2}$ and $V_{1}$ respectively so that $\left.P\right|_{V_{2} \cup V_{3}}$ and $\left.P\right|_{V_{1} \cup V_{3}}$ are free. Here are our choices for the patching isomorphisms:

for $n=2$ :

and for $n=3$ :

$$
\phi_{12}=\left[\begin{array}{ll}
z & 0 \\
l_{1} & z
\end{array}\right], \phi_{32}=\left[\begin{array}{cc}
z / l_{1} & -l_{3} \\
1 & 0
\end{array}\right], \phi_{13}=\left[\begin{array}{cc}
l_{1} & z \\
0 & l_{2}
\end{array}\right],
$$

$$
\begin{gathered}
\phi_{12}=\left[\begin{array}{ccc}
z & 0 & 0 \\
z-l_{1} & z & 0 \\
l_{1} & l_{1} & z
\end{array}\right], \phi_{32}=\left[\begin{array}{ccc}
z / l_{1} & -z / l_{1} & -l_{3} / l_{1} \\
z / l_{1}-1 & 1 & 0 \\
1 & 0 & 0
\end{array}\right], \\
\phi_{13}=\left[\begin{array}{ccc}
l_{1} & l_{1} & z \\
0 & l_{1} & z-z^{2} / l_{1} \\
0 & 0 & l_{2}
\end{array}\right] .
\end{gathered}
$$

We see that for $n=2$ and $n=3$, the 1 -cocycle identities are satisfied. The vector bundle $P$ on $V_{1} \cup V_{2} \cup V_{3}$ of rank $n$ is obtained by pasting together the local models specified above using the isomorphisms $\phi_{i j}$. It is easy to check that $\phi_{32}$ is in $G L_{n}\left(V_{2}\right)$ and $\phi_{13}$ is in $G L_{n}\left(V_{3}\right)$. This means $P$ restricts to a free module on $V_{2} \cup V_{3}$ and on $V_{1} \cup V_{3}$. We claim that $\left.P\right|_{V_{1} \cup V_{2}} \cong n J$. Note that $z$ is invertible on $V_{1} \cap V_{2}=\operatorname{Spec} R[1 / z]$, but $z$ is not a Čech 1-coboundary from $V_{1}^{*} \times V_{2}^{*}$. With respect to the open cover $\left\{V_{1}, V_{2}\right\}$ of $V_{1} \cup V_{2}$, the invertible element $z$ in $\mathcal{O}^{*}\left(V_{1} \cap V_{2}\right)$ defines a 1-cocycle in $H^{1}\left(V_{1} \cup V_{2}, \mathcal{O}^{*}\right)$ that corresponds to an invertible module $J$ on $V_{1} \cup V_{2}$ that extends to a reflexive ideal $I$ on $U_{3}$. The class of $I$ generates $\mathrm{Cl}\left(U_{3}\right)$. This can be checked as in [6, Example 3]. So the scalar matrix $\psi=1 / z$ defines a 1 -cocycle in $H^{1}\left(V_{1} \cup V_{2}, G L_{n}\right)$ and $\psi^{-1}$ corresponds to $n J$. 
Now for $n=2$ :

$$
\psi \phi_{12}=\left[\begin{array}{cc}
1 / z & 0 \\
0 & 1 / z
\end{array}\right]\left[\begin{array}{cc}
z & 0 \\
l_{1} & z
\end{array}\right]=\left[\begin{array}{cc}
1 & 0 \\
l_{1} / z & 1
\end{array}\right]=\left[\begin{array}{cc}
1 & 0 \\
\frac{z}{l_{2} l_{3}} & 1
\end{array}\right],
$$

and for $n=3$ :

$$
\psi \phi_{12}=\left[\begin{array}{ccc}
1 & 0 & 0 \\
1-l_{1} / z & 1 & 0 \\
l_{1} / z & l_{1} / z & 1
\end{array}\right]=\left[\begin{array}{ccc}
1 & 0 & 0 \\
1-\frac{z^{2}}{l_{2} l_{3}} & 1 & 0 \\
\frac{z^{2}}{l_{2} l_{3}} & \frac{z^{2}}{l_{2} l_{3}} & 1
\end{array}\right] .
$$

For $n=2$ and $n=3, \psi \phi_{12}$ is a 1 -coboundary from $V_{1}^{*} \times V_{2}^{*}$, hence $\phi_{12}$ is cohomologous to $\psi^{-1}$. So the 1-cocycle $\left\{\phi_{12}, \phi_{32}, \phi_{13}\right\}$ defines the vector bundle $P$ on $V_{1} \cup U_{2} \cup U_{3}$ of rank $n$ with the specified local models. The vector bundle $P$ extends to $X$ to give the reflexive module $M$ over $R$ of rank $n$ and $\operatorname{End}_{R}(M)$ is the desired Azumaya algebra.

Theorem 2.2. Let $R=\frac{k[x, y, z]}{\left(z^{m}-l_{1} l_{2} \cdots l_{n}\right)}$ where $m \geq 2, n \geq 3$ and $l_{1}, l_{2}, \ldots, l_{n}$ are polynomials in $k[x, y]$ satisfying

(1) for each $i<j,\left\{l_{i}, l_{j}, \frac{l_{1} l_{2} \cdots l_{n}}{l_{i} l_{j}}\right\}$ generates the unit ideal,

(2) each curve $l_{i}=0$ is nonsingular,

(3) the curve $l_{1} l_{2} \cdots l_{n}=0$ has at most nodal singularities,

(4) for each $i<j,\left\{l_{i}, l_{j}\right\}$ generates a maximal ideal.

Then for $p=2$ or 3 , the subgroup of $\mathrm{B}(K / R)$ annihilated by $p$ is generated by the classes of algebras of the form $\operatorname{End}_{R}(M)$ where $M$ is a reflexive $R$-module of rank $p$.

Proof. Let $p=2$ or 3. By [6, Example 3] $\mathrm{B}(K / R)$ is a free $\mathbb{Z} / m$-module so we assume from now on that $p \mid m$. By [6] and (5), it suffices to show that for each singular point $q$ of $X=\operatorname{Spec}(R)$, there is a reflexive module $M$ of rank $p$ such that $M_{q} \cong p I$ where $I$ is an element of order $p$ in $\mathrm{Cl}\left(R_{q}\right)$ and at every other point $q$ of $X, M_{q}$ is free. By the hypotheses (1) - (4), the singular point $q$ corresponds to a maximal ideal $\left(z, l_{i}, l_{j}\right)$ for some $i<j$. After relabeling the $l^{\prime}$ 's if necessary, let us assume $i=1$ and $j=2$. Set $g=l_{3} l_{4} \cdots l_{n}$. Let $U_{1}=\operatorname{Spec}\left(R\left[1 / l_{1}\right]\right), U_{2}=\operatorname{Spec}\left(R\left[1 / l_{2}\right]\right), U_{3}=\operatorname{Spec}(R[1 / g])$. Then $X=$ $U_{1} \cup U_{2} \cup U_{3}$. Also $\mathrm{Cl}\left(U_{3}\right) / \operatorname{Pic}\left(U_{3}\right)=\mathrm{Cl}\left(R_{q}\right) \cong \mathbb{Z} / m$. Let $V_{1}=\operatorname{Spec}\left(R\left[1 /\left(l_{2} g\right)\right]\right)$, $V_{2}=\operatorname{Spec}\left(R\left[1 /\left(l_{1} g\right)\right]\right), V_{3}=\operatorname{Spec}\left(R\left[1 /\left(l_{1} l_{2}\right)\right]\right)$. Then $V_{1} \cup V_{2}=U_{3}-q, V_{1} \cup V_{3}=$ $U_{2}$ - (finite set of points), $V_{2} \cup V_{3}=U_{1}$ - (finite set of points). We choose a 1cocycle $\left\{\phi_{12}, \phi_{13}, \phi_{32}\right\}$ with respect to the open cover $\left\{V_{1}, V_{2}, V_{3}\right\}$ so that the vector bundle it defines is free on $V_{1} \cup V_{3}$ and $V_{2} \cup V_{3}$ and such that $\phi_{12}$ defines $\left.p I\right|_{V_{1} \cup V_{2}}$. Now $V_{12}=V_{1} \cap V_{2}=\operatorname{Spec}(R[1 / z])$ and $z^{m / p} \in G L_{1}\left(V_{12}\right)$ but $z^{m / p}$ is not a coboundary from $G L_{1}\left(V_{1}\right) \times G L_{1}\left(V_{2}\right)$. We see that $z^{m / p}$ defines a 1-cocycle in $H^{1}\left(V_{1} \cup V_{2}, \mathcal{O}^{*}\right)$ that corresponds to the invertible module $\left.I\right|_{V_{1} \cup V_{2}}$. 
Our choices for the 1-cocycle are:

if $p=2$ :

$$
\phi_{12}=\left[\begin{array}{cc}
z^{m / 2} & 0 \\
l_{1} & z^{m / 2}
\end{array}\right], \phi_{32}=\left[\begin{array}{cc}
\frac{z^{m / 2}}{l_{1}} & -g \\
1 & 0
\end{array}\right], \phi_{13}=\left[\begin{array}{cc}
l_{1} & z^{m / 2} \\
0 & l_{2}
\end{array}\right],
$$

and for $p=3$ :

$$
\begin{gathered}
\phi_{12}=\left[\begin{array}{ccc}
z^{m / 3} & 0 & 0 \\
z^{m / 3}-l_{1} & z^{m / 3} & 0 \\
l_{1} & l_{1} & z^{m / 3}
\end{array}\right], \phi_{32}=\left[\begin{array}{ccc}
\frac{z^{m / 3}}{l_{1}} & -\frac{z^{m / 3}}{l_{1}} & -g / l_{1} \\
\frac{z^{m / 3}}{l_{1}}-1 & 1 & 0 \\
1 & 0 & 0
\end{array}\right], \\
\phi_{13}=\left[\begin{array}{ccc}
l_{1} & l_{1} & z^{m / 3} \\
0 & l_{1} & z^{m / 3}-\frac{z^{2 m / 3}}{l_{1}} \\
0 & 0 & l_{2}
\end{array}\right] .
\end{gathered}
$$

Note that $\phi_{32} \in G L_{2}\left(V_{2}\right), \phi_{13} \in G L_{2}\left(V_{3}\right)$ and if $p=2$ :

and for $p=3$ :

$$
\frac{1}{z^{m / 2}} \phi_{12}=\left[\begin{array}{cc}
1 & 0 \\
\frac{l_{1}}{z^{m / 2}} & 1
\end{array}\right]=\left[\begin{array}{cc}
1 & 0 \\
\frac{z^{m / 2}}{l_{2} g} & 1
\end{array}\right] \in G L_{2}\left(V_{1}\right),
$$

$$
\frac{1}{z^{m / 3}} \phi_{12}=\left[\begin{array}{ccc}
1 & 0 & 0 \\
1-\frac{l_{1}}{z^{m / 3}} & 1 & 0 \\
\frac{l_{1}}{z^{m / 3}} & \frac{l_{1}}{z^{m / 3}} & 1
\end{array}\right]=\left[\begin{array}{ccc}
1 & 0 & 0 \\
1-\frac{z^{2 m}}{l_{2} g} & 1 & 0 \\
\frac{z^{2 m}}{l_{2 g}} & \frac{z^{2 m}}{l_{2 g}} & 1
\end{array}\right] \in G L_{3}\left(V_{1}\right) .
$$

So the vector bundle $P$ defined by $\left\{\phi_{i j}\right\}$ on $V_{1} \cup V_{2} \cup V_{3}$ extends to a reflexive module $M$ on $X$ with the desired local structure. 
Example 2.3. This is another example of a normal surface, but this time we consider a toric surface with 3 singular points. We will attempt to follow the terminology and notation of [17] for toric varieties except that we prefer to denote the elements in the coordinate ring of the torus $T_{N}$ as Laurant polynomials in the indeterminates $x, y$. Let $n$ be an integer in the range $2 \ldots 5$. Let $\Delta$ be the complete fan on $\mathbb{R}^{2}$ with 2-dimensional cones $\sigma_{1}=\rho_{1}+\rho_{2}, \sigma_{2}=\rho_{2}+\rho_{3}, \sigma_{3}=\rho_{3}+\rho_{1}$ where $\rho_{1}$ is spanned by $(1,0), \rho_{2}$ is spanned by $(-1, n), \rho_{3}$ is spanned by $(-1,-n)$. Let $X=T_{N} \operatorname{emb}(\Delta)$. Then by [6, Theorem 4] we know that $\mathrm{B}(X)=\mathrm{B}(K / X)=\mathbb{Z} / n$. We proceed as in Example 2.1. Now $X$ has an open cover $\left\{U_{i}=U_{\sigma_{i}}\right\}$ corresponding to the maximal cones in $\Delta$ which splits every element of $\mathrm{B}(X)$. The idea is to build a reflexive module $M$ on $X$ using as local models, $n I(=I \oplus I \oplus \ldots \oplus I, n$ times) on $U_{1}, I$ being a generator of $\mathrm{Cl}\left(U_{1}\right)$, and on $U_{2}$ and $U_{3}$ the free module $n \mathcal{O}$. Let $V_{i}=T_{N} \operatorname{emb}\left(\rho_{i}\right)$. Then $\left\{V_{i}\right\}$ is an open cover of $X_{\text {reg }}$. We define a coherent locally free module $P$ on $X_{\text {reg }}$ of rank $n$ which extends to $M$ on $X$. From [6, Theorem 4] it follows that $H^{1}\left(X_{\text {Zar }}, \mathcal{C}\right) \cong H^{1}\left(X_{\text {ét }}, \mathcal{C}\right)$ hence from (5) it will follow that the class of $\operatorname{End}(M)$ generates $\mathrm{B}(X)$. The local models for $P$ are $n \mathcal{O}$ on each open set $V_{i}$. The patching isomorphisms $\phi_{i j}$ make up a 1-cocycle in $\check{H}^{1}\left(\left\{V_{i}\right\} / X_{\text {reg }}, G L_{n}\right)$. We choose $\phi_{12}$ so that $\left.P\right|_{V_{1} \cup V_{2}} \cong n J$ where $J$ is $I$ restricted to $\left(U_{1}\right)_{\text {reg }}=V_{1} \cup V_{2}$. We choose $\phi_{32}$ and $\phi_{13}$ so that $P$ is free of rank $n$ on $V_{2} \cup V_{3}$ and $V_{3} \cup V_{1}$. We have $V_{i}=\operatorname{Spec} k\left[\check{\rho}_{i} \cap \mathbb{Z}^{2}\right]$ and $V_{12}=V_{23}=V_{13}=\operatorname{Spec} k[x, 1 / x, y, 1 / y]$. The choices for $\phi_{i j}$ are:

for $n=2$ :

for $n=3$ :

$$
\phi_{12}=\left[\begin{array}{ll}
x & 0 \\
y & x
\end{array}\right], \phi_{32}=\left[\begin{array}{cc}
x / y & -x^{2} / y \\
1 & 0
\end{array}\right], \phi_{13}=\left[\begin{array}{ll}
y & x \\
0 & 1
\end{array}\right],
$$

$$
\phi_{12}=\left[\begin{array}{ccc}
x & 0 & 0 \\
\frac{y(x-1)}{x} & x & 0 \\
y & x & x
\end{array}\right], \phi_{32}=\left[\begin{array}{ccc}
\frac{x}{y} & -\frac{x^{2}}{y} & -\frac{x^{3}}{y} \\
\frac{x-1}{x} & 1 & 0 \\
1 & 0 & 0
\end{array}\right], \phi_{13}=\left[\begin{array}{ccc}
y & x & x \\
0 & 1 & 1-x \\
0 & 0 & 1
\end{array}\right],
$$


for $n=4$ :

$$
\begin{gathered}
\phi_{12}=\left[\begin{array}{cccc}
x & 0 & 0 & 0 \\
\frac{y\left(x^{2}-x-1\right)}{x^{2}} & x & 0 & 0 \\
y / x & 0 & x & 0 \\
y & x & x & x
\end{array}\right] \\
\phi_{32}=\left[\begin{array}{cccc}
\frac{x}{y} & -\frac{x^{2}}{y} & -\frac{x^{3}}{y} & -\frac{x^{4}}{y} \\
\frac{x^{2}-x-1}{x^{2}} & \frac{x+1}{x} & 1 & 0 \\
1 / x & -1 & 0 & 0 \\
1 & 0 & 0 & 0
\end{array}\right], \phi_{13}=\left[\begin{array}{cccc}
y & x & x & x \\
0 & 1 & 1-x & 1 \\
0 & 0 & 1 & -x \\
0 & 0 & 0 & 1
\end{array}\right],
\end{gathered}
$$

for $n=5$ :

$$
\begin{gathered}
\phi_{12}=\left[\begin{array}{ccccc}
x & 0 & 0 & 0 & 0 \\
\frac{y\left(x^{3}-x^{2}-x-1\right)}{x^{3}} & x & 0 & 0 & 0 \\
\frac{y\left(x^{2}-1\right)}{x^{2}} & x & x & 0 & 0 \\
\frac{y(x-1)}{x} & x & 0 & x & 0 \\
y & x & x & x & x
\end{array}\right] \\
\phi_{32}=\left[\begin{array}{cccccc}
\frac{x}{y} & -\frac{x^{2}}{y} & -\frac{x^{3}}{y} & -\frac{x^{4}}{y} & -\frac{x^{5}}{y} \\
\frac{x^{3}-x^{2}-x-1}{x^{3}} & \frac{x^{2}+x+1}{x^{2}} & \frac{x+1}{x} & 1 & 0 \\
\frac{x^{2}-1}{x^{2}} & \frac{1}{x} & 1 & 0 & 0 \\
\frac{x-1}{x} & & 1 & 0 & 0 & 0 \\
1 & & 0 & 0 & 0 & 0
\end{array}\right] \\
\phi_{13}=\left[\begin{array}{cccccc}
y & x & x & x & x \\
0 & 1 & 1-x & 1 & 1-x \\
0 & 0 & 1 & -x & 1-x \\
0 & 0 & 0 & 1 & 1+x \\
0 & 0 & 0 & 0 & -1
\end{array}\right] .
\end{gathered}
$$

Notice that $1 / x \phi_{12} \in G L_{n}\left(V_{2}\right)$ for each $n$. Now one checks as in Example 2.1 that these $\phi_{i j}$ 's have the properties claimed. 
Example 2.4. This is an example of a 3-dimensional normal toric variety $X$ with 3 isolated singular points satisfying $\mathrm{B}(X)=\mathrm{B}(K / X)=\mathbb{Z} / 3$. We use the same techniques as in Examples 2.1 and 2.3 to construct a reflexive module $M$ on $X$ of rank 3 such that $\operatorname{End}(M)$ generates $\mathrm{B}(X)$. Let $\Delta$ be the fan on $\mathbb{R}^{3}$ defined by the cones $\sigma_{1}=\rho_{0}+\rho_{1}+\rho_{2}, \sigma_{2}=\rho_{0}+\rho_{2}+\rho_{3}$ and $\sigma_{3}=\rho_{0}+\rho_{3}+\rho_{1}$, where $\rho_{0}=(0,0,1), \rho_{1}=(0,1,0), \rho_{2}=(3,-1,2), \rho_{3}=(-3,-4,4)$. Let $X=T_{N} \operatorname{emb}(\Delta)$. Then one can check using [7, Theorem 1$]$ that the cohomological Brauer group of $X$ is cyclic of order 3 and every element of $\mathrm{B}^{\prime}(X)$ is split by the Zariski open cover $\left\{U_{\sigma_{i}}\right\}$. From [7, Lemma 7$] H^{1}\left(X_{Z a r}, \mathcal{C}\right) \cong H^{1}\left(X_{\text {ét }}, \mathcal{C}\right)$ hence by (5) it suffices to find a reflexive module $M$ whose class under $\alpha$ has order 3 module the image of $\mathrm{Cl}(X)$. Since $\operatorname{dim} X=3$ and $X$ is non-affine, the existing methods of the literature cannot be applied directly to show that $\mathrm{B}(X)=\mathrm{B}^{\prime}(X)$. Here we give an elementary construction of a reflexive module $M$ on $X$ such that $\operatorname{End}(M)$ generates $B(X)$. It follows that $\mathrm{B}(X)=\mathrm{B}^{\prime}(X)$. Let $\Delta(1)=\left\{0, \rho_{0}, \rho_{1}, \rho_{2}, \rho_{3}\right\}$. Then $T_{N} \operatorname{emb}(\Delta(1))$ is an open subset of $X_{\text {reg }}$ and $T_{N} \operatorname{emb}(\Delta(1))$ contains all of the points of codimension 1 of $X$. It suffices therefore to construct a vector bundle $P$ on $T_{N} \operatorname{emb}(\Delta(1))$ such that $P$ extends to the desired reflexive module $M$ on $X$. As in Example 2.3, set up an open cover $\left\{V_{i}\right\}$ for $T_{N} \operatorname{emb}(\Delta(1)): V_{1}=T_{N} \operatorname{emb}\left(\left\{0, \rho_{0}, \rho_{1}\right\}\right), V_{2}=$ $T_{N} \operatorname{emb}\left(\left\{0, \rho_{0}, \rho_{2}\right\}\right), V_{3}=T_{N} \operatorname{emb}\left(\left\{0, \rho_{0}, \rho_{3}\right\}\right)$. Then check that $V_{1}^{*}=\langle x\rangle, V_{2}^{*}=$ $\left\langle x y^{3}\right\rangle, V_{3}^{*}=\left\langle x^{4} / y^{3}\right\rangle$. Also $V_{i j}=\operatorname{Spec} k[z, x, 1 / x, y, 1 / y]$ for all pairs $i j$. On $V_{12}$, the unit $y$ is not a coboundary from $V_{1}^{*} \times V_{2}^{*}$. Our local model for $P$ on $V_{1} \cup V_{2}$ is $J \oplus J \oplus J$ where $J$ is a generator for $\operatorname{Pic}\left(V_{1} \cup V_{2}\right)$. The local model for $P$ on the open sets $V_{2} \cup V_{3}$ and $V_{1} \cup V_{3}$ is the free module 30 . The choices we make for the patching isomorphisms are:

$\phi_{12}=\left[\begin{array}{ccc}y & 0 & 0 \\ \frac{x^{4}(y-1)}{y} & y & 0 \\ x^{4} & y & y\end{array}\right], \phi_{32}=\left[\begin{array}{ccc}\frac{y}{x^{4}} & -\frac{y^{2}}{x^{4}} & -\frac{y^{3}}{x^{4}} \\ \frac{y-1}{y} & 1 & 0 \\ 1 & 0 & 0\end{array}\right], \phi_{13}=\left[\begin{array}{ccc}x^{4} & y & y \\ 0 & 1 & 1-y \\ 0 & 0 & 1\end{array}\right]$.

As in Examples 2.1 and 2.3 one checks that this defines a nontrivial cocycle in $\check{H}^{1}\left(T_{N} \operatorname{emb}(\Delta(1)), G L_{3}\right)$ with the desired local structure. The sheaf $P$ extends to $M$ on $\mathrm{X}$ and $\operatorname{End}(M)$ generates $\mathrm{B}(X)=\mathrm{B}^{\prime}(X)$. 
Example 2.5. This is another example of a 3-dimensional toric variety defined by three 3-dimensional cones in $\mathbb{R}^{3}$. The only significant difference between this and Example 2.4 is that we now have three lines of singularities whereas in Example 2.4 there were only three isolated singularities. The three maximal cones are $\sigma_{1}=\rho_{0}+\rho_{1}+\rho_{2}, \sigma_{2}=\rho_{0}+\rho_{2}+\rho_{3}, \sigma_{3}=\rho_{0}+\rho_{1}+\rho_{3}$, where $\rho_{0}=(0,0,1), \rho_{1}=(1,0,1), \rho_{2}=(-1,2,1), \rho_{3}=(-1,-2,1)$. Let $\Delta$ be the fan consisting of the faces of the cones $\sigma_{1}, \sigma_{2}, \sigma_{3}$ and $X=T_{N} \mathrm{emb}(\Delta)$. One can compute $\mathrm{B}^{\prime}(X) \cong \mathbb{Z} / 2$ using the results from [7] or the procedure of Section 4. Let $\Delta(1)$ be the fan consisting of the faces of the cones $\rho_{0}, \ldots, \rho_{3}$. As in Example 2.4 set up an open cover of $T_{N} \operatorname{emb}(\Delta(1)): V_{1}=T_{N} \operatorname{emb}\left(\left\{0, \rho_{0}, \rho_{1}\right\}\right), V_{2}=T_{N} \operatorname{emb}\left(\left\{0, \rho_{0}, \rho_{2}\right\}\right)$, $V_{3}=T_{N} \operatorname{emb}\left(\left\{0, \rho_{0}, \rho_{3}\right\}\right)$. Define a 1-cocycle with respect to this open cover by:

$$
\phi_{12}=\left[\begin{array}{ll}
x & 0 \\
y & x
\end{array}\right], \phi_{32}=\left[\begin{array}{cc}
x / y & -x^{2} / y \\
1 & 0
\end{array}\right], \phi_{13}=\left[\begin{array}{ll}
y & x \\
0 & 1
\end{array}\right] .
$$

Then this defines a nontrivial reflexive module on $X$ with projective endomorphism ring.

\section{Algebras on toric varieties}

The purpose of this section is to study locally trivial Azumaya algebras on a toric variety all of whose singularities are in codimension $\leq 2$. The main result of this section is Corollary 3.4 in which we show that if $X$ is a complete toric surface and $y \in \mathrm{B}^{\prime}(X)$ has order 2 , then there is a reflexive $\mathcal{O}_{X}$-module $M$ of rank 2 such that $y$ is the Brauer class of $\operatorname{End}_{\mathcal{O}_{X}}(M)$. Along the way to proving this, our techniques allow us to construct many Azumaya algebras on toric varieties of all dimensions. We begin by proving some results on vector bundles of rank 2 on a toric variety $X=T_{N} \operatorname{emb}(\Delta)$ where $\Delta$ is the fan $\left\{0, \rho_{1}, \rho_{2}, \rho_{3}\right\}, \rho_{1}, \rho_{2}, \rho_{3}$ are distinct 1-dimensional cones in $\mathbb{R}_{N}$ and $N$ is a free $\mathbb{Z}$-lattice.

Let $V_{i}=T_{N} \operatorname{emb}\left(\left\{0, \rho_{i}\right\}\right)$. So $\mathcal{V}=\left\{V_{1}, V_{2}, V_{3}\right\}$ is an open cover of $X$. Let $U_{1}=V_{2} \cup V_{3}, U_{2}=V_{1} \cup V_{3}, U_{3}=V_{1} \cup V_{2}$ and $\delta_{i}=\left|\operatorname{Pic} U_{i}\right|$. In Proposition 3.1 below, we assume $\delta_{3}$ is even. Since Pic $U_{3}$ is cyclic, this means there is a unique invertible module $J$ on $U_{3}$ of order 2 in $\operatorname{Pic} U_{3}$. We give sufficient conditions for the existence of an indecomposable equivariant vector bundle on $X$ of rank 2 which is free on $U_{1}$ and $U_{2}$ and is isomorphic to $J \oplus J$ on $U_{3}$. Equivariant vector bundles on toric varieties have also been studied by Kaneyama [14], [13]. 
Proposition 3.1. Let $\rho_{1}, \rho_{2}, \rho_{3}$ be distinct 1 -dimensional cones in $\mathbb{R}_{N}$,

$$
\begin{array}{rlrl}
X & =T_{N} \operatorname{emb}\left(\left\{0, \rho_{1}, \rho_{2}, \rho_{3}\right\}\right), & & U_{1}=T_{N} \operatorname{emb}\left(\left\{0, \rho_{2}, \rho_{3}\right\}\right), \\
U_{2} & =T_{N} \operatorname{emb}\left(\left\{0, \rho_{1}, \rho_{3}\right\}\right), & U_{3}=T_{N} \operatorname{emb}\left(\left\{0, \rho_{1}, \rho_{2}\right\}\right) .
\end{array}
$$

Let $\delta_{i}=\mid$ Pic $U_{i} \mid$ and assume $\delta_{3}$ is even. If $\delta=\operatorname{gcd}\left(\delta_{1}, \delta_{2}, \delta_{3}\right)$ is even, then there exists an indecomposable equivariant vector bundle P of rank 2 on $X$ satisfying: $\left.P\right|_{U_{1}} \cong \mathcal{O} \oplus \mathcal{O}$, $\left.P\right|_{U_{2}} \cong \mathcal{O} \oplus \mathcal{O},\left.P\right|_{U_{3}} \cong J \oplus J$, where $J$ is the unique invertible module on $U_{3}$ of order 2. Conversely, if $\delta$ is odd, then given any such vector bundle $P$, there exists an invertible module L on $X$ such that $\left.(L \otimes P)\right|_{U_{i}} \cong \mathcal{O} \oplus \mathcal{O}$ for each $U_{i}$.

Before proving Proposition 3.1, we first establish some notation. Let $\eta_{i}$ be a primitive lattice point on $\rho_{i}$. Since $\delta_{3}$ is the greatest common divisor of the 2by-2 minors of the matrix $\left[\begin{array}{ll}\eta_{1}^{\top} & \eta_{2}^{\top}\end{array}\right]$ and we are assuming $\delta_{3}$ is even, there is a basis for $N$ with respect to which $\eta_{1}=(1,0, \ldots, 0), \eta_{2}=(a, 2 b, 0, \ldots, 0)$ and $\eta_{3}=(c, d, e, 0, \ldots, 0)$. We can assume $0<a<2 b$ and $e \geq 0$. Note that $\delta$ is even if and only if $d$ and $e$ are both even. If $e>0$, then after a transformation of the type $\left[\begin{array}{lll}1 & 0 & f \\ 0 & 1 & g \\ 0 & 0 & 1\end{array}\right]$ we can further assume $c<0$ and $d<0$

Let $N^{\prime}$ be the subgroup of $N$ spanned by $(1,0, \ldots, 0)$ and $(0,1,0, \ldots, 0)$. Then under the projection $N \rightarrow N^{\prime}, \eta_{1}, \eta_{2}, \eta_{3}$ project onto $\eta_{1}^{\prime}=(1,0), \eta_{2}^{\prime}=(a, 2 b)$ and $\eta_{3}^{\prime}=(c, d)$. The projection $N \rightarrow N^{\prime}$ induces an equivariant morphism $\pi$ : $X \rightarrow X^{\prime}=T_{N} \operatorname{emb}\left(\Delta^{\prime}\right)$ where $\Delta^{\prime}$ is the fan determined by $\rho_{i}^{\prime}=\mathbb{R}_{\geq 0} \eta_{i}^{\prime}, i=1,2,3$. Now $\pi^{*}$ is an isomorphism from Pic $U_{3}^{\prime}$ to Pic $U_{3}$. Our plan is to first prove Proposition 3.1 for $N^{\prime}$, then show that the vector bundle we construct lifts under $\pi^{*}$ to a vector bundle on $X$ with the desired attributes. The 2-dimensional results we need are summarized in the next lemma.

Lemma 3.2. Let $N=\mathbb{Z}^{2}$. Let $a, b$ be positive integers, $c$, d integers. Assume $\operatorname{gcd}(a, 2 b)=$ $\operatorname{gcd}(c, d)=1$. Assume $\rho_{1}=\mathbb{R}_{\geq 0}(1,0), \rho_{2}=\mathbb{R}_{\geq 0}(a, 2 b), \rho_{3}=\mathbb{R}_{\geq 0}(-c, d)$ are distinct cones in $\mathbb{R}_{N}$. Let $\Delta=\left\{0, \rho_{1}, \rho_{2}, \rho_{3}\right\}, X=T_{N} \operatorname{emb}(\Delta), U_{1}=T_{N} \operatorname{emb}\left(\left\{0, \rho_{2}, \rho_{3}\right\}\right)$ $U_{2}=T_{N} \operatorname{emb}\left(\left\{0, \rho_{1}, \rho_{3}\right\}\right), U_{3}=T_{N} \operatorname{emb}\left(\left\{0, \rho_{1}, \rho_{2}\right\}\right)$. If $d$ is even, then there is an equivariant vector bundle $P$ on $X$ of rank 2 such that $\left.P\right|_{U_{3}} \cong J \oplus J$, where $J$ is the invertible module on $U_{3}$ of order 2 in $\operatorname{Pic}\left(U_{3}\right),\left.P\right|_{U_{1}}$ is free and $\left.P\right|_{U_{2}}$ is free. If $d$ is odd, then there is an invertible module $P$ on $X$ such that $\left.P\right|_{U_{3}} \cong J,\left.P\right|_{U_{1}}$ is free and $\left.P\right|_{U_{2}}$ is free.

Proof. Let $V_{1}=T_{N} \operatorname{emb}\left(\left\{0, \rho_{1}\right\}\right), V_{2}=T_{N} \operatorname{emb}\left(\left\{0, \rho_{2}\right\}\right), V_{3}=T_{N} \operatorname{emb}\left(\left\{0, \rho_{3}\right\}\right)$. Then $\mathcal{V}=\left\{V_{1}, V_{2}, V_{3}\right\}$ is an open cover of $X$ and we see that $\mathcal{O}^{*}\left(V_{1}\right)=\langle y\rangle$, $\mathcal{O}^{*}\left(V_{2}\right)=\left\langle\frac{x^{2 b}}{y^{a}}\right\rangle, \mathcal{O}^{*}\left(V_{3}\right)=\left\langle\frac{x^{d}}{y^{c}}\right\rangle$, and $\mathcal{O}^{*}\left(V_{i j}\right)=\langle x, y\rangle$. As in Example 2.3, we prefer to use the indeterminate notation $x, y$ as opposed to the $e(\cdot)$ notation of [17]. The 1-cocycle in $\check{H}^{1}\left(\left\{V_{1}, V_{2}\right\} / U_{3}, \mathcal{O}^{*}\right)$ that defines $J$ is the unit $x^{b}$. 
Assume $d$ is odd. Since $d \equiv 1(\bmod 2)$, there are integers $u, v$ such that $1=$ $2 u+d v$, hence $b=(2 b) u+d(b v)$. Define $\phi_{i j}$ in $\breve{H}^{1}\left(\mathcal{V} / X, \mathcal{O}^{*}\right)$ by

$$
\phi_{12}=x^{b}, \phi_{32}=\frac{x^{b}}{y^{a u+b c v}}, \phi_{13}=y^{a u+b c v} \text {. }
$$

Clearly $\phi_{12}=\phi_{32} \phi_{13}$ and $\phi_{32}=\left(\frac{x^{2 b}}{y^{a}}\right)^{u}\left(\frac{x^{d}}{y^{c}}\right)^{b v}$ is a coboundary from $\mathcal{O}^{*}\left(V_{3}\right) \times$ $\mathcal{O}^{*}\left(V_{2}\right)$. Since $\phi_{13} \in \mathcal{O}^{*}\left(V_{1}\right)$ we see that the 1-cocycle defines an invertible module $P$ on $X$ such that $\left.P\right|_{U_{1}}$ is free and $\left.P\right|_{U_{2}}$ is free. Now $\phi_{12}$ defines the invertible module $\mathrm{J}$ on $U_{3}$ so we are done.

For the rest of the proof, assume $d$ is even. The local model for $P$ on each of $V_{1}, V_{2}, V_{3}$ is $\mathcal{O} \oplus \mathcal{O}$. It suffices to give patching isomorphisms $\phi_{i j}$ in $G L_{2}\left(V_{i j}\right)$ satisfying the 1-cocycle identity: $\phi_{12}=\phi_{32} \phi_{13}$ such that $\phi_{12}$ defines $J \oplus J$ on $V_{1} \cup V_{2}, \phi_{32}$ is a coboundary from $G L_{2}\left(V_{3}\right) \times G L_{2}\left(V_{2}\right)$ and $\phi_{13}$ is a coboundary from $G L_{2}\left(V_{1}\right) \times G L_{2}\left(V_{3}\right)$. The proof is divided into 5 cases depending on the configuration of the 1 -dimensional cones $\rho_{1}, \rho_{2}, \rho_{3}$ in $\mathbb{R}^{2}$.

Case 1. $c \leq 0$. Our choices for $\phi_{i j}$ are:

$$
\phi_{12}=\left[\begin{array}{cc}
x^{b} & 0 \\
y^{a} & x^{b}
\end{array}\right], \phi_{32}=\left[\begin{array}{cc}
x^{b} / y^{a} & -x^{2 b} / y^{a} \\
1 & 0
\end{array}\right], \phi_{13}=\left[\begin{array}{cc}
y^{a} & x^{b} \\
0 & 1
\end{array}\right] .
$$

Note that

$$
x^{-b} \phi_{12}=\left[\begin{array}{cc}
1 & 0 \\
y^{a} / x^{b} & 1
\end{array}\right]
$$

is a coboundary from $G L_{2}\left(V_{2}\right)$ since the inner product of $(-b, a)$ with $(a, 2 b)$ is $a b$ which is positive. The scalar matrix $x^{-b}$ defines $J$ on $V_{1} \cup V_{2}$ since $x^{2 b}$ is a coboundary from $V_{1}^{*} \times V_{2}^{*}$ but $x^{b}$ is not. We see that $\phi_{12}$ defines $J \oplus J$. Since $b>0$ and $y \in V_{1}^{*}$, we see that $\phi_{13}$ is a coboundary from $G L_{2}\left(V_{1}\right)$. Now

$$
\phi_{32}=\left[\begin{array}{cc}
-x^{2 b} / y^{a} & 0 \\
0 & 1
\end{array}\right]\left[\begin{array}{cc}
-1 / x^{b} & 1 \\
1 & 0
\end{array}\right] .
$$

The left factor of (7) is in $G L_{2}\left(V_{2}\right)$ since $x^{2 b} / y^{a} \in V_{2}^{*}$. The right factor in (7) is in $G L_{2}\left(V_{3}\right)$ since we are assuming $c \leq 0$. Therefore, $\phi_{32}$ is a coboundary and defines a free module on $V_{3} \cup V_{2}$ as desired.

Case 2. $c>0, d<0$. There exists a positive integer $e$ so that $-d e \geq b c$. Now set

$$
\phi_{12}=\left[\begin{array}{cc}
x^{b} & 0 \\
y^{a e} & x^{b}
\end{array}\right], \phi_{32}=\left[\begin{array}{cc}
\frac{x^{b}}{y^{a+e}} & -\frac{x^{2 b}}{y^{a}} \\
1 & 0
\end{array}\right], \phi_{13}=\left[\begin{array}{cc}
y^{a+e} & x^{b} \\
0 & y^{-e}
\end{array}\right] .
$$


First note that $\phi_{12}=\phi_{32} \phi_{13}$ and $\phi_{13} \in G L_{2}\left(V_{1}\right)$. Now

$$
\phi_{32}=\left[\begin{array}{cc}
-\frac{x^{2 b}}{y^{a}} & 0 \\
0 & 1
\end{array}\right]\left[\begin{array}{cc}
-\frac{1}{x^{b} y^{e}} & 1 \\
1 & 0
\end{array}\right] .
$$

The left factor in (8) is in $G L_{2}\left(V_{2}\right)$. The right factor of (8) is in $G L_{2}\left(V_{3}\right)$ since the inner product of $(-b,-e)$ with $(c, d)$ is $-b c-e d \geq 0$. Therefore $\phi_{13}$ and $\phi_{32}$ define free modules on $U_{2}$ and $U_{1}$ respectively. We have the factorization

$$
\phi_{12}=\left[\begin{array}{cc}
1 & 0 \\
\frac{y^{a e}}{x^{b}} & 1
\end{array}\right]\left[\begin{array}{cc}
x^{b} & 0 \\
0 & x^{b}
\end{array}\right],
$$

and the left factor of $(9)$ is in $G L_{2}\left(V_{2}\right)$ since the inner product of $(-b, a e)$ with $(a, 2 b)$ is $-a b+2 a b e=a b(2 e-1) \geq 0$. So $\phi_{12}$ defines $J \oplus J$ on $U_{3}$.

Case 3. $0<d<c, 0<a<2 b$. Set

$$
\begin{gathered}
\phi_{12}=\left[\begin{array}{cc}
x^{b(d-1)} & 0 \\
y^{b(d-1)} & x^{b}
\end{array}\right], \phi_{32}=\left[\begin{array}{cc}
\left(\frac{x}{y}\right)^{b(d-1)} & -\left(\frac{x^{d}}{y^{c}}\right)^{b} \\
1 & 0
\end{array}\right], \\
\phi_{13}=\left[\begin{array}{cc}
y^{b(d-1)} & x^{b} \\
0 & y^{b(c-d+1)}
\end{array}\right] .
\end{gathered}
$$

First note that $\phi_{12}=\phi_{32} \phi_{13}$ and $\phi_{13} \in G L_{2}\left(V_{1}\right)$. Now $\phi_{32} \in G L_{2}\left(V_{3}\right)$ since $x / y \in \Gamma\left(V_{3}, \mathcal{O}\right)$. This is because the inner product of $(1,-1)$ and $(c, d)$ is $c-d>0$. Now

$$
\phi_{12}=\left[\begin{array}{cc}
\left(\frac{x^{2 b}}{y^{a}}\right)^{(d-2) / 2} & 0 \\
\frac{y^{b(d-1)-a(d-2) / 2}}{x^{b}} & 1
\end{array}\right]\left[\begin{array}{cc}
x^{b} & 0 \\
0 & x^{b}
\end{array}\right]\left[\begin{array}{cc}
y^{a(d-2) / 2} & 0 \\
0 & 1
\end{array}\right],
$$

the right factor in (10) is in $G L_{2}\left(V_{1}\right)$ and the left factor is in $G L_{2}\left(V_{2}\right)$ since the inner product of $(-b, b(d-1)-a(d-2) / 2)$ with $(a, 2 b)$ is $-a b+2 b^{2}(d-1)-a b(d-2)$ $=b(d-1)(2 b-a)>0$. Therefore $\phi_{12}$ is cohomologous to the scalar matrix $x^{b}$ on $U_{3}$, hence defines $J \oplus J$.

Case 4. $0<c<d, 0<a<2 b$ and $2 b c>a d$. There exists a positive integer $e$ such that

$$
2 b e(2 b c-a d)+b(a-2 b)>0
$$


Set

$$
\begin{gathered}
\phi_{12}=\left[\begin{array}{cc}
x^{2 b e d-b} & 0 \\
y^{2 b e c-b} & x^{b}
\end{array}\right], \phi_{32}=\left[\begin{array}{cc}
\left(\frac{x^{d}}{y^{c}}\right)^{2 b e}\left(\frac{y}{x}\right)^{b} & -\left(\frac{x^{d}}{y^{c}}\right)^{2 b e} \\
1 & 0
\end{array}\right], \\
\phi_{13}=\left[\begin{array}{cc}
y^{2 b e c-b} & x^{b} \\
0 & y^{b}
\end{array}\right] .
\end{gathered}
$$

First note that $\phi_{12}=\phi_{32} \phi_{13}$ and $\phi_{13} \in G L_{2}\left(V_{1}\right)$. Since $\frac{x^{d}}{y^{c}} \in \mathcal{O}^{*}\left(V_{3}\right)$ and the inner product of $(-1,1)$ with $(c, d)$ is $d-c>0, \phi_{32} \in G L_{2}\left(V_{3}\right)$. We have the factorization

$$
\phi_{12}=\left[\begin{array}{cc}
\left(\frac{x^{2 b}}{y^{a}}\right)^{e d-1} & 0 \\
\frac{y^{2 b e c-b-a e d+a}}{x^{b}} & 1
\end{array}\right]\left[\begin{array}{cc}
x^{b} & 0 \\
0 & x^{b}
\end{array}\right]\left[\begin{array}{cc}
y^{a(e d-1)} & 0 \\
0 & 1
\end{array}\right],
$$

Since the inner product of $(-b, 2 b e c-b-a e d+a)$ with $(a, 2 b)$ is $-a b+2 b e(2 b c-$ $a d)+2 b(a-b)$ which is positive by the choice of $e$ in (11), it follows that the left hand factor of (12) is in $G L_{2}\left(V_{2}\right)$. The right hand factor of (12) is in $G L_{2}\left(V_{1}\right)$ so $\phi_{12}$ defines $J \oplus J$ on $U_{3}$.

Case 5. $0<c<d, 0<a<2 b$ and $2 b c<a d$. In this case, $\rho_{2}$ and $\rho_{3}$ are both in the first quadrant of $\mathbb{R}^{2}$ and $\rho_{2}$ is in the 2-dimensional cone spanned by $\rho_{1}$ and $\rho_{3}$. That is, $\eta_{2}=r \eta_{1}+s \eta_{3}$ for some pair of positive rational numbers $r$, s. We can pick a suitable change of basis matrix $T \in G L_{2}(\mathbb{Z})$ such that $\eta_{2} T=\eta_{2}^{\prime}=(1,0)$, $\eta_{1} T=\eta_{1}^{\prime}=\left(a^{\prime}, 2 b^{\prime}\right)$ and $\eta_{3} T=\eta_{3}^{\prime}=\left(c^{\prime}, d^{\prime}\right)$ where $0<a^{\prime}<2 b^{\prime}, d^{\prime} \equiv 0(\bmod 2)$. Since $(1,0)=\eta_{2}^{\prime}=r \eta_{1}^{\prime}+s \eta_{3}^{\prime}$ with $r$ and $s$ positive, we see that $\eta_{3}^{\prime}$ must be in the third or fourth quadrant. So we are in either Case 1 or Case 2.

Proof of Proposition 3.1. We return to the notation of the paragraph immediately following the statement of the proposition. Assume first that $e>0$ and $\operatorname{rank} N>$ 2. Let $V_{i}=T_{N} \operatorname{emb}\left(\left\{0, \rho_{i}\right\}\right)$. Then under the projection $\pi$, the ring $\mathcal{O}\left(V_{i}^{\prime}\right)$ embeds into the ring $\mathcal{O}\left(V_{i}\right)$. The configuration of the fan $\Delta^{\prime}$ corresponds to Case 1 of the proof of Lemma 3.2 and the cocycle $\phi_{i j}^{\prime}$ in $\check{H}^{1}\left(\mathcal{V}^{\prime} / X^{\prime}, \mathcal{O}^{*}\right)$ maps under $\pi^{*}$ to a cocycle in $\check{H}^{1}\left(\mathcal{V} / X, \mathcal{O}^{*}\right)$ that defines an equivariant vector bundle $P$ with the correct local structure. The fact that $P$ is indecomposable when $\delta$ is even follows from Proposition 3.3 below because $P$ extends to give a reflexive $\mathcal{O}_{\tilde{X}}{ }^{-}$ module $M$ such that $\operatorname{End}_{\mathcal{O}_{\tilde{X}}}(M)$ has order 2 in $\mathrm{B}^{\prime}(K / \tilde{X})$. Here we denote by $\tilde{X}$ the toric variety obtained from adding to the fan $\Delta$ the three 2-dimensional cones $\rho_{1}+\rho_{2}, \rho_{2}+\rho_{3}, \rho_{1}+\rho_{3}$. Now if $\delta$ is even and either $e=0$ or $\operatorname{rank} N=2$, we use another method to show that $P$ is indecomposable. In this case we simply project a fan onto $\Delta$ from a higher dimension. The fan $\Delta$ is the projection of the fan $\Sigma$ determined by the 3 cones spanned by $(1,0, \ldots, 0),(a, 2 b, 0, \ldots, 0)$ and $(c, d, 2,0, \ldots, 0)$ in $\mathbb{R}_{N} \times \mathbb{R}$. By our previous argument, $P$ lifts to an indecomposable vector bundle on the higher dimensional variety defined by $\Sigma$, hence is indecomposable on $X$. 
Now assume $\delta$ is odd. Then by Lemma 3.2 there is a rank 1 vector bundle $L$ on $X$ which is isomorphic to $J$ on $U_{3}$, is free on $U_{1}$ and is free on $U_{2}$. So if $P$ is a rank 2 vector bundle as in the statement of the proposition, then $L \otimes P$ is free on the open cover $\left\{U_{1}, U_{2}, U_{3}\right\}$.

Now we consider a toric variety $X=T_{N} \mathrm{emb}(\Delta)$ such that all of the cones in $\Delta$ have dimension $\leq 2$. By [9, Theorem 2.9] the elements of order $\leq 2$ in the relative cohomological Brauer group $\mathrm{B}^{\prime}(X)$ form an elementary 2-group of rank $\leq e$ where $e$ is the number of edges in the graph $\Gamma$ of $\Delta$ that are not in a 2maximal spanning tree of $\Gamma$. The graph $\Gamma$ is defined as follows. Let $\tau_{1}, \ldots, \tau_{m}$ be the 2-dimensional cones and $\rho_{1}, \ldots, \rho_{n}$ the 1 -dimensional cones of $\Gamma$. These cones make up the vertex set of $\Gamma$. An edge connects $\rho_{i}$ and $\tau_{j}$ if and only if $\rho_{i}$ is a face of $\tau_{j}$. The graph $\Gamma$ is edge-weighted by setting the 2-weight of the edge $\rho_{i-} \tau_{j}$ to be the 2-adic valuation of the order of the finite cyclic group $\mathrm{Cl}\left(U_{\tau_{j}}\right)$ where $U_{\tau_{j}}=$ $T_{N}$ emb $\left(\Delta\left(\tau_{j}\right)\right)$, and $\Delta\left(\tau_{j}\right)$ is the fan consisting of $\tau_{j}$ and its faces. Theorem 2.6 of [9] says that if the graph $\Gamma$ consists of just 1 cycle, then ${ }_{2} \mathrm{~B}^{\prime}(K / X)$ is cyclic. The order of ${ }_{2} \mathrm{~B}^{\prime}(K / X)$ in this case is 2 if and only if each class group $\mathrm{Cl}\left(U_{\tau_{j}}\right)$ has even order. In the next proposition we show that in this case the group ${ }_{2} \mathrm{~B}^{\prime}(K / X)$ is generated by the class of an Azumaya algebra, hence ${ }_{2} \mathrm{~B}(K / X)={ }_{2} \mathrm{~B}^{\prime}(K / X)$.

Proposition 3.3. Let $\Delta$ be a fan on $\mathbb{R}_{N}$ and let $X=T_{N} \operatorname{emb}(\Delta)$. Assume all of the cones in $\Delta$ have dimension $\leq 2$ and that the 2-dimensional faces $\tau_{1}, \ldots, \tau_{m}$ and 1-dimensional faces $\rho_{1}, \ldots, \rho_{n}$ of $\Delta$ can be ordered so that $\tau_{i} \cap \tau_{i+1}=\rho_{i+1}(1 \leq i \leq m-1)$ and $\tau_{m} \cap \tau_{1}=\rho_{1}$. If the order of $\mathrm{Cl}\left(U_{\tau_{i}}\right)$ is even for each $i$, then the element of order 2 in ${ }_{2} \mathrm{~B}^{\prime}(K / X)$ is the class of End $_{\mathcal{O}_{X}}(M)$ for some reflexive $\mathcal{O}_{X}$-module $M$ of rank 2.

Proof. There is a unique cycle $\rho_{1} \tau_{1} \rho_{2} \rho_{2} \ldots \tau_{m} \rho_{1}$ in the graph $\Gamma$ of $\Delta$. The cones $\rho_{m+1}, \ldots, \rho_{n}$ (if they exist) are not faces of any $\tau_{j}$. Let $V_{i}=U_{\rho_{i}}$ for $1 \leq i \leq n$. Then $V=\left\{V_{1}, \ldots, V_{n}\right\}$ is an open cover for $X_{r e g}$. We show that there exists a vector bundle $P$ on $X_{\text {reg }}$ split by $V$ such that $\left.P\right|_{V_{1} \cup V_{2}} \cong J \oplus J$ where $J$ is the unique invertible module of order 2 on $V_{1} \cup V_{2},\left.P\right|_{V_{i} \cup V_{i+1}} \cong \mathcal{O} \oplus \mathcal{O}$ for $2 \leq i \leq m-1$, and $\left.P\right|_{V_{m} \cup V_{1}} \cong \mathcal{O} \oplus \mathcal{O}$. Then $P$ will extend to a reflexive module $M$ on $X$ such that $\left.M\right|_{U_{\tau_{1}}} \cong I \oplus I$ where $I$ is the element of order 2 in $\operatorname{Cl}\left(U_{\tau_{1}}\right)$, and $\left.M\right|_{U_{\tau_{i}}}$ is free for $2 \leq i \leq m$. By [7, Lemma 7$] H^{1}\left(X_{\text {Zar }}, \mathcal{C}\right) \cong H^{1}\left(X_{\text {ét }}, \mathcal{C}\right)$. It follows from [9, Theorem 2.3] that $H^{0}\left(X_{Z a r}, \mathcal{P}\right) \cong \underset{i=1}{m} \mathrm{Cl}\left(U_{\tau_{i}}\right)$. Therefore by [9, Theorem 2.6] and by (5) it follows that $\operatorname{End}_{\mathcal{O}_{X}}(M)$ is an Azumaya algebra whose class generates ${ }_{2} \mathrm{~B}^{\prime}(K / X)$. 
From Proposition 3.1 there is a vector bundle on $V_{1} \cup V_{2} \cup V_{3}$ defined by a 1-cocycle $\left\{\phi_{12}, \phi_{32}, \phi_{13}\right\}$ where $\phi_{32}$ and $\phi_{13}$ are coboundaries. Hence there are $\alpha_{i}, \beta_{i} \in G L_{2}\left(V_{i}\right)$ such that $\phi_{32}=\alpha_{2} \alpha_{3}$ and $\phi_{13}=\beta_{3} \beta_{1}$. We have $\phi_{12}=\phi_{32} \phi_{13}$ so $\left(\phi_{12} \beta_{1}^{-1}\right)=\left(\alpha_{2} \alpha_{3} \beta_{3}\right)\left(\beta_{1} \beta_{1}^{-1}\right)$ and we can assume $\phi_{13}=1$. Now we show how to extend $\left\{\phi_{12}, \phi_{32}, \phi_{13}\right\}$ to the open cover $\left\{V_{1}, \ldots, V_{n}\right\}$ of $X_{\text {reg. }}$. Proceed recursively, to define:

$$
\begin{gathered}
\phi_{43}=1, \phi_{42}=\phi_{32} \phi_{43}, \phi_{41}=1, \\
\phi_{54}=1, \phi_{53}=1, \phi_{52}=\phi_{32} \phi_{53}, \phi_{51}=1,
\end{gathered}
$$

and for an arbitrary $i$ between 4 and $n$,

$$
\phi_{i, i-1}=1, \ldots, \phi_{i 3}=1, \phi_{i 2}=\phi_{32} \phi_{i 3}, \phi_{i 1}=1 .
$$

We see that this defines a 1 -cocycle $\left\{\phi_{i j}\right\}$ in $\check{H}^{1}\left(\mathcal{V} / X_{\text {reg }}, \mathcal{O}^{*}\right)$ and that the vector bundle $P$ defined by $\left\{\phi_{i j}\right\}$ has the desired local structure. The proposition follows.

Corollary 2.9 of [9] showed that if $X$ is a complete toric surface, then $\mathrm{B}(K / X)=$ $\mathrm{B}(X)=\mathrm{B}^{\prime}(X)$ is cyclic. The proof relied on the Hoobler-Gabber theorem to show $\mathrm{B}(X)=\mathrm{B}^{\prime}(X)$. Our next result shows every element of order 2 in $\mathrm{B}^{\prime}(X)$ is the class of an explicit Azumaya algebra $\operatorname{End}_{\mathcal{O}_{X}}(M)$ such that $\operatorname{rank}(M)=2$.

Corollary 3.4. Let $\Delta$ be a complete fan on $\mathbb{R}^{2}$ and $X=T_{N} \mathrm{emb}(\Delta)$ the associated toric surface. If $\mathrm{B}(X) \cong \mathbb{Z} / 2 n$, then there is a reflexive $\mathcal{O}_{X}$-module $M$ on $X$ with $\operatorname{rank} M=2$ such that $\operatorname{End}_{\mathcal{O}_{X}}(M)$ is a nontrivial Azumaya algebra representing the class of order 2 in $\mathrm{B}(X)$.

Proof. This follows immediately from Proposition 3.3.

\section{Computing the cohomological Brauer group of a toric Variety}

The purpose of this section is to show how one might compute the étale cohomology groups $H^{p}\left(X_{\text {ét }}, \mathbb{G}_{m}\right)$ in degrees $p=0,1$ and 2 of a toric variety $X$ with coefficients in the sheaf of units. The method is to reduce the computation down to the problem of diagonalizing a matrix with integral coefficients. The procedure outlined in this article has been fully implemented by the author as a program written in the " $\mathrm{C}$ " programming language.

The groups that we want to compute are finitely generated abelian groups. Our method for computing them is to reduce the problem to a matrix theory computation involving matrices with integer coefficients. Let us begin with a brief description of the algorithms for matrices with integer coefficients which are to be used.

Let $S$ be an $m \times n$ matrix with integer coefficients. The basic procedure that we perform on $S$ is "diagonalization", or equivalently, "put $S$ into Smith normal form." This means that we find invertible matrices $X$ and $Y$ so that XSY = $\operatorname{diag}\left\{d_{1}, d_{2}, \ldots, d_{s}, 0, \ldots, 0\right\}$ where $d_{1}\left|d_{2}\right| \cdots \mid d_{s}$ and $d_{s} \neq 0$. Thus $S$ has column rank $s$. An algorithm for computing $X, Y$ and $d_{1}, \ldots, d_{S}$ can be found in [5]. 
Once we have computed the matrix $Y$ we can find simultaneous bases for the column space of $S$ and $\mathbb{Z}^{m}$. By this we mean a set of vectors $\left\{x_{1}, \ldots, x_{s}\right\}$ that extend to a basis for $\mathbb{Z}^{m}$ and such that a basis for the column space of $S$ is $\left\{d_{1} x_{1}, d_{2} x_{2}, \ldots, d_{s} x_{s}\right\}$. We simply take $\left\{x_{1}, \ldots, x_{s}\right\}$ to be the columns of the matrix

$$
X^{-1} \operatorname{diag}\{1,1, \ldots, 1,0, \ldots, 0\} \text { (where there are } s \text { ones) }
$$

$$
=S Y \operatorname{diag}\left\{d_{1}^{-1}, d_{2}^{-1}, \ldots, d_{s}^{-1}, 0, \ldots, 0\right\} \text {. }
$$

The vectors $\left\{x_{1}, \ldots, x_{s}\right\}$ span the smallest direct summand of $\mathbb{Z}^{m}$ that contains the column space of $S$. A basis for the kernel of $S$ exists in the columns numbered $s+1, \ldots, n$ of the matrix $Y$.

Say the columns of $B$ contain a basis for a direct summand of rank $s$ of $\mathbb{Z}^{m}$ and $X$ is an invertible matrix such that $X B=\operatorname{diag}\{1, \ldots, 1,0, \ldots, 0\}$. Let $A$ be an $m \times l$ matrix whose columns are in the column space of $B$. The matrix $X A$ represents the function which maps the columns of $A$ into the column space of $B$.

Suppose we have an $m \times l$ matrix $A$ and an $n \times m$ matrix $B$ such that $B A=0$ and we want to compute the homology group $\operatorname{ker} B / \operatorname{im} A$. First find a basis for the kernel of $B$ as above. Say this basis makes up the columns of the matrix $K$. Find invertible matrices $X$ and $Y$ so that $X K Y=\operatorname{diag}\{1, \ldots, 1\}$. We want to write the columns of $A$ as linear combinations of the columns of $K Y$. The matrix for the embedding columnspace $(A) \rightarrow \operatorname{columnspace}(K Y)$ is the $\operatorname{rank}(K) \times l$ matrix $X A$. The invariant factors of $\operatorname{ker} B / \operatorname{im} A$ are obtained by diagonalizing this matrix.

We now discuss how to use the above algorithms to compute the cohomological Brauer group of a toric variety. Let $N=\mathbb{Z}^{r}, M=\operatorname{Hom}_{\mathbb{Z}}(N, \mathbb{Z})$. Let $\Delta$ denote a fan on $N_{\mathbb{R}}=\mathbb{R}^{r}$ and $X=T_{N} \operatorname{emb}(\Delta)$ the corresponding toric variety over the algebraically closed field $k$ of characteristic 0 . Let $K$ denote the function field of $X$. The cohomological Brauer group of $X$ is the second étale cohomology group with coefficients in the sheaf of units, $H^{2}\left(X_{\text {ét }}, G_{m}\right)$. According to [7, Theorem 1$]$, if $\tilde{X}$ is an equivariant desingularization of $X$, then there is a split-exact sequence with natural maps

$$
0 \rightarrow H^{2}\left(K / X_{\text {ét }}, G_{m}\right) \rightarrow H^{2}\left(X_{\text {ét }}, G_{m}\right) \rightarrow H^{2}\left(\tilde{X}_{\text {ét }}, G_{m}\right) \rightarrow 0 .
$$

Sequence (13) reduces the calculation of $H^{2}\left(X_{\text {ét }}, G_{m}\right)$ down to the computation of the 2 smaller groups in the sequence. The relative cohomological Brauer group $H^{2}\left(K / X_{\text {ét }}, G_{m}\right)$ consists of the 2-cocycles which are generically split. The group $H^{2}\left(\tilde{X}_{\text {ét }}, \mathbb{G}_{m}\right)$ is naturally isomorphic to the image of the Brauer group of $X$ in the Brauer group of the function field of $X, \mathrm{~B}(K)$. In order to compute these groups, our method allows us to also compute the zeroth and first degree étale cohomology groups without much extra work. The divisor class group is the Picard group of a suitable open subset of $X$ and we might as well compute it as well. The groups which we plan to compute are now enumerated.

(1) The image of the Brauer group of $X$ in $B(K)$. This group is naturally isomorphic to the Brauer group $\mathrm{B}(\tilde{X})=H^{2}\left(\tilde{X}_{\text {ét }}, \mathrm{G}_{m}\right)$ of an equivariant desingularization $\tilde{X}$ of $X$.

(2) The divisor class group $\mathrm{Cl}(X)$.

(3) The group of units $H^{0}\left(X_{\text {ét }}, G_{m}\right)$. 
(4) The Picard group $\operatorname{Pic}(X)=H^{1}\left(X_{\text {ét }}, \mathbb{G}_{m}\right)$.

(5) The relative cohomological Brauer group, $H^{2}\left(K / X, \mathbb{G}_{m}\right)$.

Let $\left\{\rho_{1}, \ldots, \rho_{n}\right\}=\Delta(1)$ be the cones in $\Delta$ of dimension 1. For each $\rho_{i} \in \Delta(1)$ choose a primitive generator $\eta_{i} \in \mathbb{Z}^{r}$ such that $\rho_{i}=\mathbb{R}_{\geq 0} \cdot \eta_{i}$.

Let $\tilde{X}$ be an equivariant desingularization of $X$. Then $\tilde{X}$ is the toric variety associated to a fan $\Delta^{\prime}$ obtained by subdividing $\Delta$. Any maximal cone $\tau \in \Delta^{\prime}$ is contained in some maximal cone $\sigma \in \Delta$. For such a pair $(\tau, \sigma)$, the group generated by $\tau \cap N$ is equal to the smallest direct summand of $N$ that contains $\{\rho \in \Delta(1) \mid \rho \subseteq \sigma\}$. Let $N^{\prime}$ be the group generated by $\cup_{\tau \in \Delta^{\prime}} \sigma \cap N$. The invariant factors of $N / N^{\prime}$ can be computed using the methods described above. For each maximal cone $\sigma$ of $\Delta$, find a basis $L(\sigma)$ for the smallest direct summand of $N$ containing $\left\{\eta_{i} \mid \rho_{i} \subseteq \sigma\right\}$. Set up a matrix whose columns are the elements of $\cup_{\sigma \in \Delta} L(\sigma)$ and compute the invariant factors of this matrix using the above mentioned methods. Say the invariants are $a_{1}, \ldots, a_{r}$. In order to compute group 1 above, we use [9, Theorem 1.1] which says $H^{2}\left(\tilde{X}_{\text {ét }}, \mathbb{G}_{m}\right)$ is isomorphic to $\oplus_{i=1}^{r-1} \operatorname{Hom}\left(\mathbb{Z} / a_{i}, \mathbb{Q} / \mathbb{Z}\right)^{r-i}$. Note that $\operatorname{Hom}\left(\mathbb{Z} / a_{i}, \mathbb{Q} / \mathbb{Z}\right)=0, \mathbb{Z} / a_{i}, \mathbb{Q} / \mathbb{Z}$ according to whether $\left|a_{i}\right|=1,\left|a_{i}\right|>1, a_{i}=0$.

The divisor class group of $X$ is isomorphic to that of the toric variety associated to the fan $\left\{0, \rho_{1}, \ldots, \rho_{n}\right\}$. The class group of the latter variety is the cokernel of the function $M \rightarrow \oplus_{i=1}^{n} \mathbb{Z} \cdot \rho_{i}$. The rows of the matrix for this function are just the transposed vectors $\eta_{1}, \ldots, \eta_{n}$. So the class group is determined using the methods from above.

As in [7, sequence (11)], let $\mathcal{S F}$ denote the sheaf of support functions on $\Delta, \mathcal{M}$ the constant sheaf $M$ and $U$ the sheaf kernel defined by the sequence

$$
0 \rightarrow \mathcal{U} \rightarrow \mathcal{M} \rightarrow \mathcal{S F} \rightarrow 0
$$

It follows from [17] that $\operatorname{Pic}(X) \cong \operatorname{SF}(\Delta) / \operatorname{im}(M)$ and from [7, Theorem 1.a] that $H^{2}\left(K / X_{\text {ét }}, G_{m}\right) \cong \breve{H}^{1}(\Delta, \mathcal{S F})$. Let $\mathcal{L}$ denote the cokernel of the morphism $\mathcal{U} \rightarrow \mathcal{M}$ in the category of presheaves. So $\mathcal{L}$ is a presheaf which is locally isomorphic to $\mathcal{S F}$. That is, $\mathcal{S F}$ is the sheaf associated to $\mathcal{L}$. For any cone $\sigma \in \Delta$, let $\Delta(\sigma)$ denote the subfan of $\Delta$ consisting of $\sigma$ and all of its faces. Then $\mathcal{L}(\Delta(\sigma))=\mathcal{S F}(\Delta(\sigma))$ since support functions on a cone are linear. Therefore we see that $\check{H}^{p}(\Delta, \mathcal{S F})=$ $\breve{H}^{p}(\Delta, \mathcal{L})$ for all $p$. If $\left\{\sigma_{1}, \ldots, \sigma_{m}\right\}$ are the maximal cones of $\Delta$, and $\sigma_{i j}$ denotes $\sigma_{i} \cap \sigma_{j}$, then the Čech complex

$$
0 \rightarrow \bigoplus_{i} \mathcal{L}\left(\Delta\left(\sigma_{i}\right)\right) \stackrel{\delta^{0}}{\longrightarrow} \bigoplus_{i<j} \mathcal{L}\left(\Delta\left(\sigma_{i j}\right)\right) \stackrel{\delta^{1}}{\longrightarrow} \bigoplus_{i<j<k} \mathcal{L}\left(\Delta\left(\sigma_{i j k}\right)\right)
$$

can be used to compute the groups $H^{p}(\Delta, \mathcal{S F})=H^{p}(\Delta, \mathcal{L})$. The sequence

$$
0 \rightarrow \mathcal{U}(\Delta(\sigma)) \rightarrow M \rightarrow \mathcal{L}(\Delta(\sigma)) \rightarrow 0
$$

is exact, so we see that $\mathcal{L}(\Delta(\sigma))$ is just the dual of the group $N \cap \mathbb{R} \cdot \sigma$. Given any cone $\sigma \in \Delta$, we find $\mathcal{L}(\Delta(\sigma))$ as follows. First set up a matrix $S$ whose columns consist of those $\eta_{i}$ such that $\rho_{i} \in \sigma$. A basis $L\left(\sigma_{i}\right)$ for $\mathcal{L}(\Delta(\sigma))$ is computed by methods of the introduction by finding a basis for the smallest direct summand of $\mathbb{Z}^{r}$ containing the column space of $S$. For this and other computations involving $L(\sigma)$ it is not necessary to distinguish between $N$ and its dual $M$. If $\tau$ is a face of $\sigma$, there is a projection $\mathcal{L}(\Delta(\sigma)) \rightarrow \mathcal{L}(\Delta(\tau))$. The matrix for this projection 
T. J. FORD

corresponds to writing the elements in the basis $L(\tau)$ in terms of the basis $L(\sigma)$ hence can be carried out using the above algorithms. The ingredients for writing the matrix for $\delta^{0}$ and $\delta^{1}$ are now available. Each is just a suitably interpreted direct sum of projections of the form $\mathcal{L}(\Delta(\sigma)) \rightarrow \mathcal{L}(\Delta(\tau))$.

The kernel of $\delta^{0}$ is the group of support functions $\operatorname{SF}(\Delta)$. As mentioned above, the Picard group is computed as $\operatorname{SF}(\Delta) / \operatorname{im}(M)$. The kernel of the map $M \rightarrow$ $\operatorname{SF}(\Delta)$ is $\mathcal{U}(\Delta)$ which is just $H^{0}\left(X_{\text {ét }}, \mathrm{G}_{m}\right) / k^{*}$ hence we can compute the group of units on $X$. Again as mentioned above, the relative Brauer group $H^{2}\left(K / X_{\text {ét }}, \mathbb{G}_{m}\right)$ is the first homology group $\operatorname{ker}\left(\delta^{1}\right) / \operatorname{im}\left(\delta^{0}\right)$.

\section{REFERENCES}

[1] B. Auslander, Central separable algebras which are locally endomorphism rings of free modules, Proc. Amer. Math. Soc. 30 (1971), 395-404.

[2] Bernice Auslander, The Brauer group of a ringed space, J. Algebra 4 (1966), 220-273. MR 33 \#7362

[3] H. Bass and R. Guralnick, Projective modules with free multiples and powers, Proc. Amer. Math. Soc. 96 (1986), 207-208.

[4] Hyman Bass, Algebraic K-theory, W. A. Benjamin, Inc., New York-Amsterdam, 1968. MR 40 \#2736

[5] Charles W. Curtis and Irving Reiner, Representation theory of finite groups and associative algebras, Interscience Publishers, a division of John Wiley \& Sons, New York-London, 1962. MR 26 \#2519

[6] F. R. DeMeyer and T. J. Ford, Nontrivial, locally trivial Azumaya algebras, Azumaya algebras, actions, and modules (Bloomington, IN, 1990), Amer. Math. Soc., Providence, RI, 1992, pp. 39-49. MR 93a:13001

[7] F. R. DeMeyer, T. J. Ford, and R. Miranda, The cohomological Brauer group of a toric variety, J. Algebraic Geom. 2 (1993), no. 1, 137-154. MR 93i:14051

[8] F. R. DeMeyer and K. Regnier, Examples of reflexive modules, Methods in Module Theory (Colorado Springs, CO, 1991), Lecture Notes in Pure and Appl. Math., vol. 140, Dekker, New York, 1993, pp. $67-73$.

[9] Frank R. DeMeyer and Timothy J. Ford, On the Brauer group of toric varieties, Trans. Amer. Math. Soc. 335 (1993), no. 2, 559-577. MR 93d:14077

[10] Alexander Grothendieck, Le groupe de Brauer I, II, III, Dix Exposés sur la Cohomologie des Schémas, North Holland, Amsterdam, 1968, pp. 46-188.

[11] Robin Hartshorne, Algebraic geometry, Springer-Verlag, New York, 1977. MR 57 \#3116

[12] Raymond T. Hoobler, When is $\operatorname{Br}(X)=\operatorname{Br}^{\prime}(X)$ ?, Brauer groups in ring theory and algebraic geometry (Wilrijk, 1981), Springer, Berlin, 1982, pp. 231-244. MR 83g:14006

[13] T. Kaneyama, On equivariant vector bundles on an almost homogeneous variety, Nagoya Math. J. 57 (1975), 65-86.

[14] , Torus-equivariant vector bundles on projective spaces, Nagoya Math. J. 111 (1988), 25-40.

[15] H. Lee and M. Orzech, Brauer groups, class groups and maximal orders for a Krull scheme, Canad. J. Math. 34 (1982), 996-1010.

[16] James S. Milne, Étale cohomology, Princeton University Press, Princeton, N.J., 1980. MR 81j:14002

[17] Tadao Oda, Convex bodies and algebraic geometry, Springer-Verlag, Berlin, 1988. MR 88m:14038

[18] M. Ojanguren, A nontrivial, locally trivial algebra, J. Algebra 29 (1974), 510-512.

Department of Mathematics, Florida Atlantic University, Boca Raton, Florida 33431

E-mail address: Ford@fau.edu 